\title{
Allowing Short-Lived TCP Sessions to Ramp-UP in Broadband Wireless Networks
}

\author{
Toktam Mahmoodi, Vasilis Friderikos, Hamid Aghvami \\ Centre for Telecommunications Research, King's College London, \\ Strand, London WC2R 2LS, UK \\ \{toktam.mahmoodi, vasilis.friderikos, hamid.aghvami\}@kcl.ac.uk
}

\begin{abstract}
In this paper, we propose a dynamic OFDMA based subcarrier/power allocation which aims to protect the newly established TCP connections. The proposed allocation utilizes an estimation of the current congestion window of TCP connections to allocate resources so that vulnerable flows with small congestion windows avoid entering packet recovery via retransmission time out. In addition, for the long-lived TCP flows, the theoretical TCP throughput which can be accomplished in their end-to-end path is used as their desired achievable data rate on wireless link. Such a technique can be of significant importance since due to its popularity, TCP is commonly used over the broadband wireless networks.
\end{abstract}

\section{INTRODUCTION}

As the Internet grows both in terms of the number of users and diversity of applications, providing fair and efficient allocation of the available network resources becomes increasingly challenging. The Transmission Control Protocol (TCP) is the default transport layer protocol used in the Internet to provide reliable end-to-end communications. TCP carries $95 \%$ of today's Internet traffic and $80 \%$ of the total number of the flows in the Internet among which a large majority are short-lived flows [1][2]. However, TCP exhibits a number of shortcomings when the underlying wireless medium deviates from the reliability of the wired medium for which TCP was originally designed to serve [3].

A new TCP connection initializes in slow start phase, where the congestion window (cwnd) is small and packet loss can be a dramatic event. The occurrence of a a packet loss in the newly established TCP connection, can lead to the occurrence of the Retransmission Time Out (RTO), causing delay to the data transmission in the order of seconds. The repetition of such an event exponentially increases the RTO due to the TCP back-off, resulting in a large aggregate delay at the very outset of the session, which may cause the user to prematurely terminate the session. On the other hand, in the slow start phase cwnd is small, thus a TCP flow is not sensitive to the allocated data rate.

Unlike long-lived TCP connections, the performance of short-lived connections is highly dependent on the slow start phase. Therefore, various approaches are discussed in the literature to either shorten the slow start phase or improve the performance of TCP in this phase. For example, "QuickStart" for TCP [4] allows TCP to capture its desired sending rate by quickly increasing its cwnd. Another research work presented in [5] utilizes the cross-layer information on the available bandwidth to increase its cwnd accordingly in the slow start phase. In all these works, a bottom-up approach is used. Alternatively, we advocate the use of a top-down approach as well, i.e., the resource allocation scheme at the wireless link-layer protects the TCP flows in the slow start phase. This not only allows for the performance of shortlived TCP connections to be increased, but also provides an opportunity for the newly established TCP connections to grow their cwnd and to utilize the available wireless bandwidth.

After crossing a certain threshold, a TCP flow will enter the congestion avoidance phase, where it requires sufficient resources in order to maintain its cwnd and further increase its data rate. In this phase, where the cwnd is larger, the main constraint is the allocated wireless channel to the corresponding TCP flow, even with the expense of higher error rates, since low channel rate inevitably results in packet loss due to congestion. The TCP-aware channel allocation algorithm in CDMA networks proposed in [6] adapts the channel rate in response to the TCP sending rate. Another approach presented in [7], providing extra protection for TCP flows that have a cwnd smaller than the network bandwidth-delay product by using more robust modulation or higher transmission power for them.

To this end, with respect to the evolution of TCP cwnd, we define a state diagram for each TCP flow such that being in any state requires a different level of protection, and data rate. Thus, a TCP-aware resource allocation algorithm for OFDMA-based access networks is proposed that adapts its target Bit Error Rate (BER) and minimum required data rate per flow according to the state of the corresponding flow. This resource allocation scheme attempts firstly, to protect the newly established TCP connections, and secondly to provide a more balanced performance towards TCP throughput that is not only an optimal resource allocation for each TCP flow, but also is a fairer distribution among TCP flows. The data rate constraint is based on the estimated current cwnd of TCP for short-lived TCP connections, and on the closed form expression of TCP steady state throughput [8] for the longlived TCP connections. In addition, we study the performance of the proposed method, for various combination of short-lived and long-lived TCP flows and extensive simulation results are presented. Although all competing end-to-end flows in the investigations of this work are TCP flows, other end-to-end flows such as UDP can also be assumed among the active 
flows.

This paper is organized as follows. Section II presents the related state of the art. In Section III we detail the system model and baseline assumptions used in the paper. In section IV, the proposed TCP-aware allocation problem is introduced, together with the associated techniques to solve the corresponding optimization problems. After investigating the performance of the proposed scheme, Section V presents a heuristic approach to provide real-time solution for the resource allocation problem. Finally, this paper concludes in Section VI.

\section{BACKGROUND STUDY}

TCP is the default transport layer protocol in the Internet and is commonly used over wireless networks. However, TCP does not perform well when the underlying medium is wireless that is characterized by random losses and intermittent connectivity. To this end, a body of research has evolved over the past few years that addresses TCP-aware resource allocation algorithms at the wireless link-layer aiming to enhance the performance of the end-to-end TCP flows.

TCP-aware resource allocation algorithms over a CDMA network are studied in [6], where the objective is to maximize the throughput. The proposed algorithm in that paper, adapts the channel rate in response to the TCP sending rate, allowing it to trade off channel rate and the error rate in a TCP-friendly manner. A joint congestion control and power allocation scheme for CDMA based wireless networks is proposed in [9], which is based on a generalized network utility maximization framework. In the context of IEEE 802.16, reference [10] proposes a TCP-aware allocation algorithm that estimates the bandwidth demand based on the long-term data rate, and allocates resources accordingly. We present a TCP-aware resource allocation scheme in OFDMA-based wireless networks in [11] that provides proportional data rate among the end-to-end TCP flows.

In this work, our focus is on the the OFDMA-based wireless networks. Orthogonal Frequency Division Multiple Access (OFDMA) is specified by the new generations of wireless networks such as Long Term Evolution (LTE) [12] and IEEE 802.16e [13] as their access method. This access technique divides the available bandwidth into multiple orthogonal subcarriers, allowing users to transmit simultaneously through allocating different subsets of the available subcarriers to different users. Therefore, the implied problem of subcarrier allocation, and transmission power and rate distribution among users has been a prominent area of research over the past few years. To this end, objectives such as maximizing the overall data rate subject to power or BER constraints, which is more relevant for data centric networks, have been studied [14]. Other formulations do, however, consider fairness, either by prioritization using the weighted sum rate method [15], or by introducing proportional rate constraints [16]. Despite the extensive investigations in this area, aspects as pertain to the end-to-end data transmission perspective have not been sufficiently addressed.

\section{System MOdeL}

We assume there are $n$ active TCP flows, all in congestion avoidance phase and there are $m$ subcarriers available at the base station. Given $r_{i j}$ the data rate of user $i$ in subcarrier $j$, and assuming a single TCP flow per user, the achievable rate for user $i$ can be written as follows,

$$
R_{i}=\sum_{j=1}^{m} a_{i j} r_{i j}
$$

$$
a_{i j}= \begin{cases}1 & \text { if subcarrier } j \text { is assigned to user } i, \\ 0 & \text { if subcarrier } j \text { is not assigned to user } i .\end{cases}
$$

Data rate allocation for each user is based on adaptive modulation and the associated BER for each subcarrier is expressed based on the adaptive M-array quadratic modulation (M-QAM) [17]. Given $\gamma_{i j}$, the $i$ th user's received SNR on subcarrier $j$, and $b_{i j}$ the corresponding BER, it can be expressed as follows,

$$
b_{i j} \approx c_{1} e^{-c_{2} \frac{\gamma_{i j}}{2^{r_{i j}}-1}},
$$

where $c_{1} \approx 0.2, c_{2} \approx 1.5$. Denoting by $G_{i j}$ the channel gain of user $i$ on the subcarrier $j$, by $p_{i j}$ the allocated power to flow $i$ on the subcarrier $j$, and by $\sigma^{2}$, the thermal noise power, the corresponding SNR is given in Equation (4),

$$
\gamma_{i j}=\frac{p_{i j} G_{i j}}{\sigma^{2}}
$$

Solving for $r_{i j}$, the achievable rate for user $i$ on the $j$ th subcarrier can be described with Equation (5) as follows,

$$
r_{i j}=w_{j} \log _{2}\left(1+\frac{c_{2} p_{i j} G_{i j}}{-\sigma^{2} \ln \left(b_{i j} / c_{1}\right)}\right) \text { bits } / s .
$$

In Equation (5), $w_{j}$ is the bandwidth of subcarrier $j$ which is assumed to be equal for all subcarriers and will be denoted hereafter by $w$. Without loss of generality, a slow-fading channel is assumed such that the channel is constant within each OFDM frame. The slowly time varying assumption is crucial since we also ascertain that perfect estimation of the subcarriers is available for each user. Moreover, mobile users and the base station are synchronized, thus there is no intercarrier interference.

\section{A. TCP Flows and their State Diagram}

As mentioned earlier, TCP establishes its connection in slow start phase with its cwnd equal to one Maximum Segment Size (MSS), thus transmits one segment. Upon receiving the ACKnowledgement (ACK) for this segment, the cwnd is doubled, and the next transmission is performed-the time takes from sending a packet to the return of its corresponding ACK is called Round Trip Time (RTT). If packet loss occurs in one of the first two windows, receiver can not generate duplicate ACK. Thus, sender TCP can only detect the loss via timer expiration, which poses a large delay to the data transmission. Packet loss that occurs in the third window can be detected via duplicate ACK, hence more protections should be applied to the delivery of the packets in the first two 


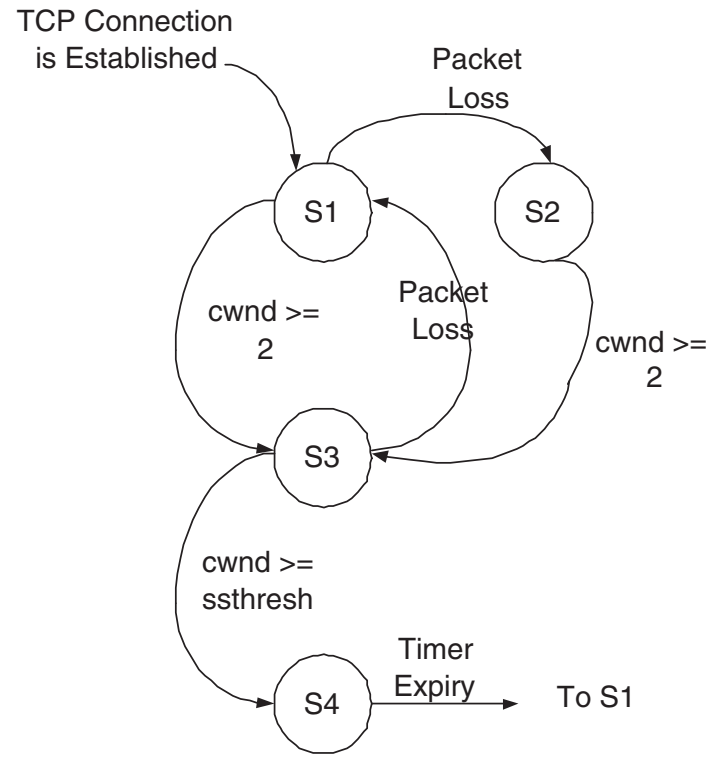

Figure 1. State of each TCP flow is changed based on its cwnd and the occurrence of loss at the initial stage of its connection.

windows, which can be translated to the lower target BER on the wireless channel utilized by these TCP flows. Afterwards, when cwnd reaches the slow start threshold (ssthresh), TCP connection transits to congestion avoidance phase and slows down the increase rate of cwnd. In the congestion avoidance phase, higher rate on the wireless channel is required even with the expense of higher error rate since low channel rate inevitably results in packet loss due to congestion.

To this end, we define a state diagram for each TCP flow, with respect to the evolution of the TCP cwnd, such that being in any state requires different level of protection-target $\mathrm{BER}$ - and data rate. This state diagram captures the three levels of TCP congestion window size and the occurrence of loss when the cwnd is smaller than two, in four states. Figure 1 demonstrates this state diagram, in which $S_{1}$ represents the establishment of a new TCP connection where cwnd is equal to one. If loss occurs while in $S_{1}$, the transition to $S_{2}$ performs where the protection level increases. By increasing the cwnd to the larger value than two, the state transits to $S_{3}$. Finally as cwnd reaches ssthresh, transition to $S_{4}$ will occur. In the event of loss that is detected via timer expiry, TCP re-enters the slow start phase and restarts its cwnd to one, thus $S_{4}$ transits to $S_{1}$.

Given $S=\left\{S_{1}, S_{2}, S_{3}, S_{4}\right\}$ the four states that each TCP flow can reside, the set of target BERs for each flow can be defined accordingly, $\mathrm{BER}_{\text {target }}=\left\{\lambda_{1}, \lambda_{2}, \lambda_{3}, \lambda_{4}\right\}$ such that $\lambda_{2} \leq \lambda_{1} \leq \lambda_{3} \leq \lambda_{4}$. The target $B E R$ at each state can be accomplished by adaptive M-array quadratic modulation (MQAM) [17].

\section{B. Achievable Data Rate by TCP Flows}

As discussed earlier, allocated subcarriers and power to user $i$ defines the achievable data rate for that user over the wireless link. On the other hand, the theoretical end-to-end throughput bounds the steady state data rate of each TCP flow. We note that the end-to-end TCP throughput is not only affected by the BER of the channel, but also by the RTT.

The steady state throughput can only be computed for the long-live TCP flows. These TCP flows reside in state $S_{4}$, thus they have the same target BER and the diversity among their throughput mainly arises from the diversity among their RTTs. TCP throughput of flow $i$ is expressed as a closed-form function proportional to the probability of a packet in error, $e_{i}$, and the end-to-end RTT [8]. The simple term that can express this function for the long-lived TCP flows in Packets/s, is as follows,

$$
B_{i}=\frac{1}{R T T_{i}} \cdot \sqrt{\frac{3}{2 e_{i}}}
$$

Assuming that wireless link is the bottleneck, the probability of a TCP packet in error, $e_{i}$, can be computed from the wireless link BER. The $\overline{R T T}$ is the smooth value of RTT, which suppresses the instantaneous variation in the RTT.

The throughput of newly established TCP connections can be computed from their cwnd, and is expressed by cwnd/RTT. In order to calculate TCP throughput at the wireless base station, the information of TCP's RTT and cwnd are required. TCP timing information can be included in the Timestamp option of the TCP segment [18]. Moreover, various methods are presented in the literature to estimate RTT passively at any router in the middle of the end-to-end pathsuch as wireless access point [19]. The research work in [20], presents range of solutions to estimate the cwnd of TCP at any middle router of the end-to-end path.

\section{TCP-AWARE Resource Allocation Problem}

Common optimization goal would be to determine the users'transmission functions $[A]_{i j}=a_{i j}$ and power matrix $[P]_{i j}=p_{i j}$ in order to maximize the overall rate with regard to the power constraints. In addition, we constrain the minimum achievable rate and the maximum BER for each flow in a TCP-aware fashion.

As discussed earlier, TCP flows with small cwnd require a more reliable channel (with low BER), but flows with larger cwnd require more transmission rate on the wireless link. Given the set of target BERs, BER target $=\left\{\lambda_{1}, \lambda_{2}, \lambda_{3}, \lambda_{4}\right\}$, the minimum required rate for each TCP flow $i$, denoted by $\eta_{i}$, can be computed based on its theoretical achievable throughput. For newly established TCP connections, this throughput can be calculated using their cwnd, and for long-lived TCP connections the steady state throughput of Equation (6) can be used. Therefore, $\eta_{i}$ in Packets/s is formulated as follows,

$$
\eta_{i}= \begin{cases}\frac{c w n d_{i}}{R T T_{i}}, & \text { if flow } i \in\left\{S_{1}, S_{2}, S_{3}\right\}, \\ B_{i}, & \text { if flow } i \in\left\{S_{4}\right\} .\end{cases}
$$

Assuming that we can achieve the same BER on all the allocated subcarriers to the TCP flow $i, b_{i}$ denotes the achievable BER by this flow. Therefore, our resource allocation problem can be formulated as follows,

$$
\text { (P) : Maximize } \sum_{i=1}^{n} \sum_{j=1}^{m} a_{i j} w \log _{2}\left(1+\frac{c_{2} p_{i j} G_{i j}}{-\sigma^{2} \ln \left(b_{i} / c_{1}\right)}\right) \text {, }
$$




$$
\begin{aligned}
\text { subject to: } b_{i} \leq \lambda_{k}, & \forall i \in S_{k} \\
R_{i} \geq \eta_{i}, & \forall i \in\{1, \ldots, n\} \\
\sum_{i=1}^{n} \sum_{j=1}^{m} a_{i j} p_{i j} \leq p_{t}, & \\
\sum_{i=1}^{n} a_{i j} \leq 1, & \forall j \in\{1, \ldots, m\} \\
p_{i j} \geq 0, & \forall i \in\{1, \ldots, n\}, j \in\{1, \ldots, m\} \\
a_{i j} \in\{0,1\} . & \forall i \in\{1, \ldots, n\}, j \in\{1, \ldots, m\}
\end{aligned}
$$

Constraint (10) restricts the total allocated power to the maximum available power at the base station, $p_{t}$. Constraint (11) ensures that every subcarrier is assigned to only one user.

This problem address a mixed integer programming problem which pose a high computational complexity. To solve the problem optimally, discrete problem $(\mathrm{P})$ can be reformulated to a continuous optimization problem. Thus, constraint (13) can be rewritten such that as $a_{i j}$ bounded in $[0,1]$. Moreover, the objective function also needs to be rewritten as,

$$
\left(\mathrm{P}^{\prime}\right) \text { : Maximize } \sum_{i=1}^{n} R_{i}-M \cdot \sum_{i=1}^{n} \sum_{j=1}^{m} a_{i j}\left(1-a_{i j}\right) .
$$

where $M$ is a relatively large value to ensure the integer assignment for $a_{i j}$.

\section{Performance Investigations}

We solve the continuous formulation of our proposed resource allocation problem using TOMLAB solver. In order to investigate the performance of the proposed scheme, we consider a number of different scenarios. An OFDMA system with 52 subcarriers (equal to the number of OFDM subcarriers in IEEE 802.11a) is simulated. The rest of simulation parameters are similar to the ones used in [13]. The available bandwidth is $5 \mathrm{MHz}$, maximum power at the base station is $40 \mathrm{dBm}$, and the average value of SNR is $20 \mathrm{~dB}$. The thermal noise power, $\sigma^{2}$, is $-107 \mathrm{dBm}$-Johnson-Nyquist noise over $5 \mathrm{MHz}$ bandwidth-, and the set of target BERs is $\left\{10^{-5}, 10^{-6}, 5 \cdot 10^{-5}, 10^{-4}\right\}$.

The MSS is set to the standard maximum transfer unit of an Ethernet network which is $1460 \mathrm{~B}$, and the ssthresh is 8 MSS. We further assume that the end-to-end RTT for any of the TCP flows is a uniformly distributed random variable in the range [10 ms, $200 \mathrm{~ms}$ ]. We perform 500 Monte Carlo simulations, while each simulation round runs with a new set of random RTT values per TCP flow, and a new set of random SNR values per subcarrier.

\section{A. Performance Observations}

Given different combinations of short-lived and long-lived TCP flows, two simulation scenarios are studied where the total of 20 active TCP flows are assumed. In the first simulation scenario it is assumed that at the initial simulations time, $50 \%$ of the TCP flows are in one of the first three states and the other $50 \%$ are at the congestion avoidance phase. The second simulation scenario changes this balance towards the newly established TCP flows such that $70 \%$ of TCP flows are initialized in slow start and the other $30 \%$ in congestion avoidance.

For the TCP flows in slow start at the initial point of simulation, cwnd is a uniformly distributed random variable between one and eight, which means they reside either in $S_{1}$ or $S_{3}$. Moving to $S_{2}$ is the result of packet loss while residing in $S_{1}$. Simulation results are compared with the resource allocation scheme that have a single target BER of $10^{-4}$ and no minimum rate constraint.

From the initial time of simulation, each TCP flow lasts for five RTTs, and the number of TCP flows that transit from slow start to congestion avoidance phase during the simulation is studied. Note that if the cwnd is equal to two at the initial simulation time, depending on the location of loss, more than two packet loss results in the failure in transiting to the congestion avoidance.

Simulation results that presented in Figure 2 show the percentage of TCP flows that transit to congestion avoidance versus their cwnd at the initial point of the simulation for the two scenarios. The average percentage of this transition for the benchmark problem is approximately $35 \%$, while using our proposed scheme up to $70 \%$ of TCP flows can alter from slow start to congestion avoidance.

Further observation from this figure show that, using the set of target BER, as we defined, none of the TCP flows with cwnd equal to one or two could transit to congestion avoidance within five RTTs. Thus, simulation scenario three is performed to investigate the minimum number of RTTs that are required for this transition. In the third simulation scenario, from 20 active TCP flows at the initial point of simulation, 10 have cwnd equal to one and the other 10 have cwnd equal to two.

Results presented in Figure 3 show that, given the initial cwnd one or two, how many RTTs are needed for the corresponding TCP flow to transit to congestion avoidance phase - their cwnd increases to a value larger than eight MSS. These results, which are presented over 500 runs of the simulation, show that the average number of required RTTs are approximately 8 using our proposed scheme, although this number is increased to 12 RTTs for the benchmark.

As discussed earlier, to accomplish the target BER for each individual TCP flow, using the adaptive M-QAM is assumed. Therefore, it is expected that the aggregated data rate will be decreased. On the other hand, TCP flows with small cwnd do not require high dat rate. In other words, even if high data rate is allocated on the wireless channel, they can not achieve the allocated rate over the end-to-end path due to their small cwnd. Hence, we are speculating that the decrease in the aggregated data rate is not significant. To this end, the fourth simulation scenario is performed to study the aggregated throughput among TCP flows.

In the simulation scenario four, there are ten active TCP flows such that their cwnd at the initial point of simulation is a uniformly distributed random variable between one and 15 . If the cwnd is larger than eight, it is assumed to be in congestion avoidance phase and its TCP throughput is calculated using 


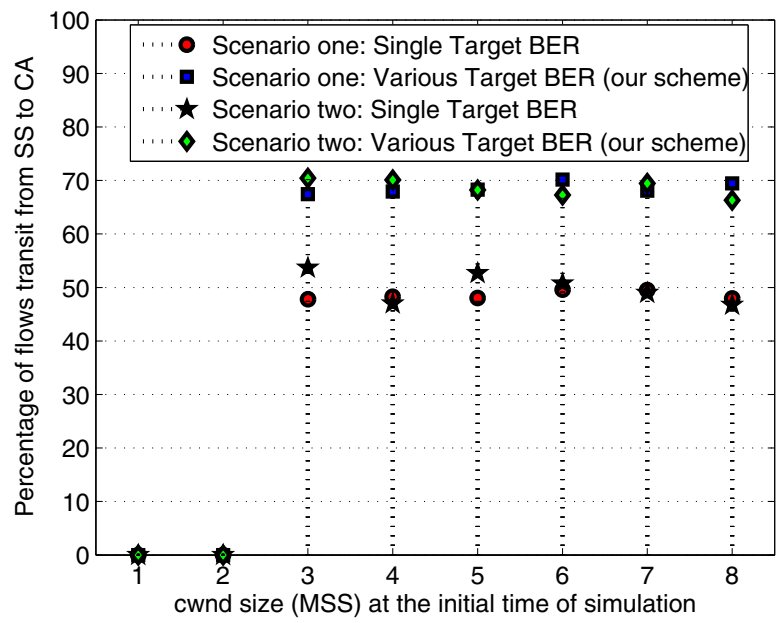

Figure 2. Simulation Scenario one and Two: The percentage of TCP flows that can transit from Slow Start (SS) to Congestion Avoidance (CA).

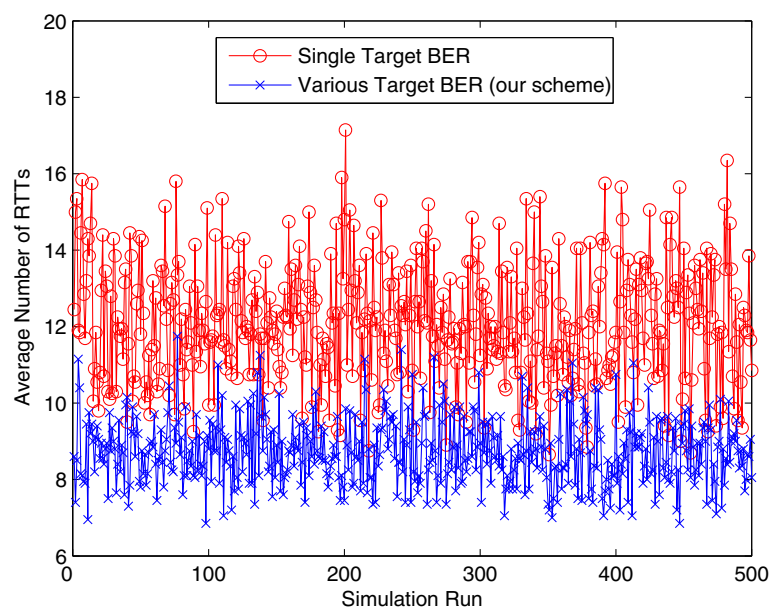

Figure 3. Simulation Scenario Three: Minim number of RTT that is needed for the initial cwnd of one/two to transit to Congestion Avoidance (CA).

Equation (6). Results of this simulation scenario show that, using our scheme, aggregated throughput is decreased by $1.5 \%$ in average. Figure 4 shows the minimum, maximum and average throughput as achieved by the ten users in the five rounds of RTT. In this figure red boxes show the achieved throughput by the benchmark scheme having single target BER in each RTT from the initial point of the TCP connections, and the blue boxes are related to our scheme with various target BER values. In addition to the evolution of TCP throughput at each RTT, it can be seen that the minimum achieved throughput is improved in our scheme, which is resulted from the minimum rate constraint (9).

\section{B. Real-Time Solution (Suboptimal)}

Although problem $\left(\mathrm{P}^{\prime}\right)$ can be solved using well-known optimization techniques, it is prohibitive for the base station to solve this problem in real time due to its complexity. Thus, we

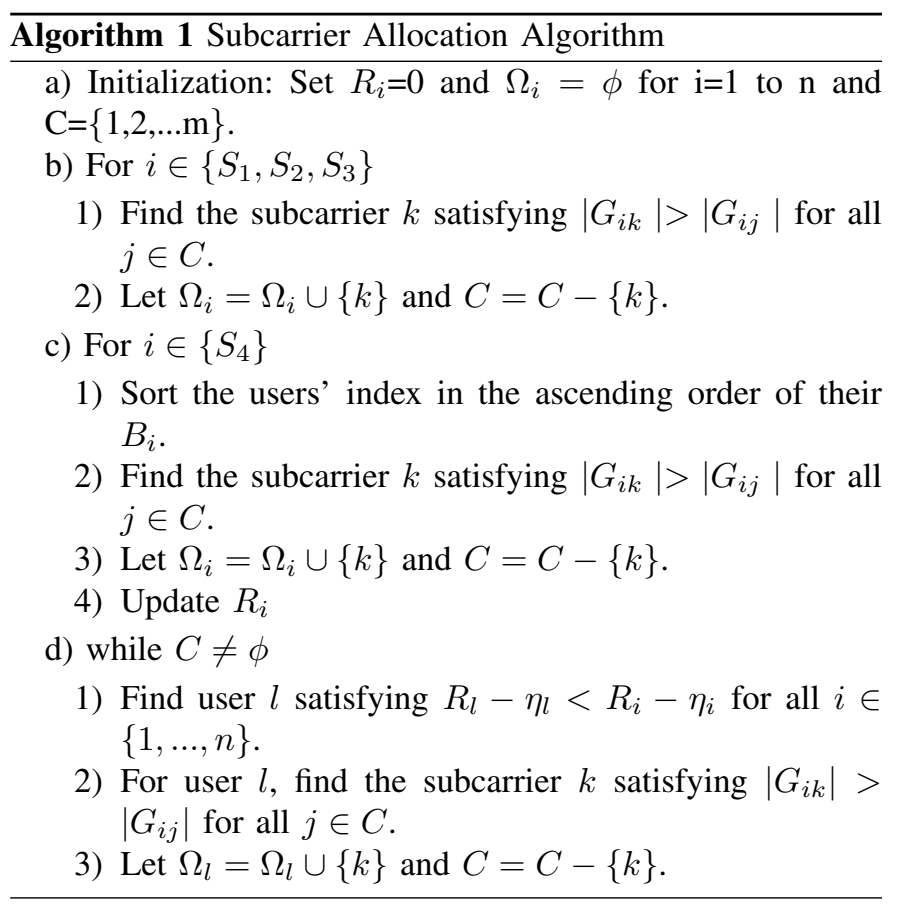

present a heuristic that can provide a real-time solution, which is also suboptimal. The addressed optimization problem can be decoupled to the two separate problems. Firstly to allocate the subcarriers, and secondly distribute power to a certain set of the allocated subcarriers. The first problem itself is an NPhard combinatorial optimization problem with finite number of combinations, which can also be more complex when number of users are increased.

Similar approach to [16] is taken to decouple the optimization problem $(\mathrm{P})$. In the subcarrier allocation it is assumed that power is equally distributed in all the subcarriers, therefore the solution is suboptimal. We detail a suboptimal algorithm, where primarily TCP flows that are in $\left\{S_{1}, S_{2}, S_{3}\right\}$ are allocated subcarriers (step (b)). Secondly, the remainder of TCP flows (flows in $S_{4}$ ) are allocated in few iterations. For the first iteration, these flows are sorted in the order of their steady state TCP throughput (step (c)), and from the next iteration, the flows that have not yet satisfied constraint (9) are further allocated (step (d)). This algorithm is detailed in Algorithm 1, in which $\Omega_{i}$ is the set of assigned subcarriers to user $i$, such that $\Omega_{i_{1}}$ and $\Omega_{i_{2}}$ are mutually exclusive if $i_{1} \neq i_{2}$.

In the next step, and based on a pre-defined subcarrier allocation, problem $(\mathrm{P})$ is simplified into a maximization problem over continuous variables $p_{i j}$.

$$
\left(\mathrm{P}^{\prime \prime}\right) \text { : Maximize } \sum_{i=1}^{n} \sum_{j \in \Omega_{i}} w \log _{2}\left(1+\frac{c_{2} p_{i j} G_{i j}}{-\sigma^{2} \ln \left(b_{i} / c_{1}\right)}\right)
$$

The reformulated Problem $\left(\mathrm{P}^{\prime \prime}\right)$ can be solved using the lagrangian dual function. Differentiating the lagrangian dual function with respect to $p_{i j}$ and set the derivative to zero, power can be distributed similar to [21][16]. 


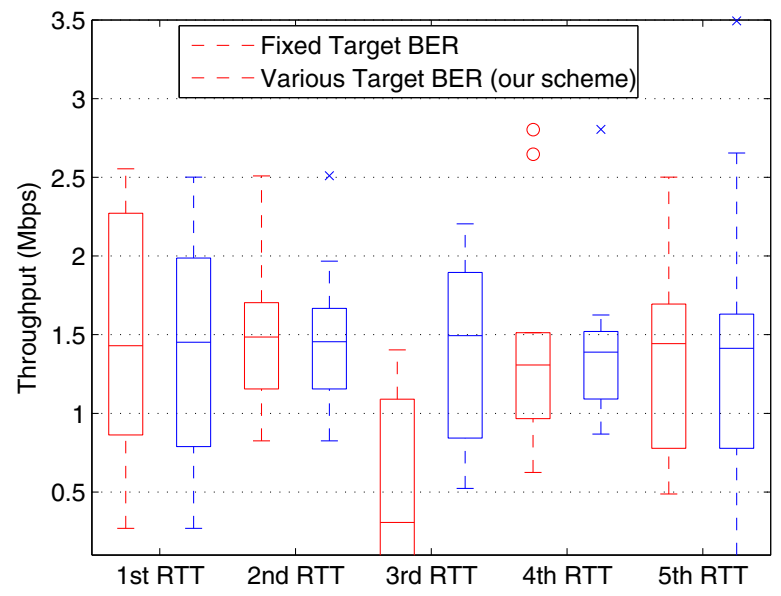

Figure 4. Throughput (Mbps) as achieved by the ten TCP flows in the five rounds of RTT; the first box is throughput as achieved by the benchmark problem, and the second box is throughput as achieved using our scheme.

\section{CONCLUSIONS}

In this paper, a TCP-aware resource allocation technique is proposed so that vulnerable flows with small congestion window avoid entering packet recovery via retransmission time out. This aim is accomplished by setting lower target BER for such TCP flows in the actual resource allocation scheme at the wireless link-layer. The rational behind this work is the existence of large number of short-lived TCP connections over the mobile broadband networks, which their performance is highly dependent on the performance of their slow start phase. On the other hand, for the long-lived TCP connections, the main constraint is the allocated data rate even with the expense of higher error rates, since low channel rate inevitably results in packet loss due to congestion.

The formulation of this resource allocation technique is presented in OFDMA-based wireless networks, where performance investigations are carried out. Simulation results reveal that the percentage of TCP flows that can transit from slow start phase to congestion avoidance phase increases significantly. Moreover, the average number of RTTs that is required for newly established TCP connections to transit from slow start to congestion avoidance phase is decreased. Despite the above mentioned achievements, the aggregated throughput is slightly decreased.

\section{ACKNOWLEDGMENT}

The work reported in this paper has formed part of the Delivery Efficiency Core Research Programme of the Virtual Centre of Excellence in Mobile \& Personal Communications, Mobile VCE, www.mobilevce.com. This research has been funded by EPSRC and by the Industrial Companies who are Members of Mobile VCE. Fully detailed technical reports on this research are available to Industrial Members of Mobile VCE.

\section{REFERENCES}

[1] S. Ebrahimi-Taghizadeh, A. Helmy, and S. Gupta, "TCP vs. TCP: a Systematic Study of Adverse Impact of Short-lived TCP Flows on Longlived TCP Flows," Proc. IEEE INFOCOM'05, Miami, USA, Mar. 2005.

[2] W. John and S. Tafvelin, "Analysis of Internet Backbone Traffic and Header Anomalies observed," Proc. ACM SIGCOMM, pp. 111-116, Kyoto, Japan, Aug. 2007.

[3] X. Chen, H. Zhai, J. Wang, and Y. Fang, "A survey on improving TCP performance over wireless networks," Springer Net. Theory \& Appl. Series, vol. 16, pp. 657-695, 2005.

[4] S. Floyd, M. Allman, A. Jain, and P. Sarolahti, "Quick-Start for TCP and IP," IETF RFC 4782, 2007.

[5] W. K. Chai and G. Pavlou, "Cross-Layer Enhancement to TCP SlowStart over Geostationary Bandwidth on Demand Satellite Networks," Proc. NEW2AN'2007, Sep. 2007.

[6] M. Ghaderi, A. Sridharan, H. Zang, D. Towsley, and R. Cruz, "TCPAware Channel Allocation in CDMA Networks," IEEE Trans. Mobile Comput., vol. 8, pp. 14-28, Jan. 2009.

[7] J. P. Singh, Y. Li, N. Bambos, A. Bahai, B. Xu, , and G. Zimmermann, "TCP Performance Dynamics and Link-Layer Adaptation Based Optimization Methods for Wireless Networks," IEEE Trans. Wireless Commun., vol. 6, pp. 1864-1879, May 2007.

[8] J. Padhye, V. Firoiu, D. Towsley, and J. Kurose, "Modeling TCP Throughput: A Simple Model and its Empirical Validation," Proc. ACM SIGCOMM '98, pp. 303-314, Vancouver, Canada, Sept. 1998.

[9] M. Chiang, "Balancing Transport and Physical Layers in Wireless Multihop Networks: Jointly Optimal Congestion Control and Power Control," IEEE J Sel. Areas Commun., vol. 23, pp. 104-116, Jan. 2005.

[10] S. Kim and I. Yeom, "TCP-aware Uplink Scheduling for IEEE 802.16," IEEE Commun. Letters, vol. 11, pp. 146-148, Feb. 2007.

[11] T. Mahmoodi, V. Friderikos, O. Holland, and A. H. Aghvami, "TCPaware Resource Allocation Problem in OFDMA based Wireless Networks," Proc. Int'l Workshop Cross-Layer Design (IWCLD), Palma, Spain, Jun. 2009

[12] "IEEE Std. 802.16e-2005: Air Interface for Fixed and Mobile Broadband Wireless Access Systems, Physical and Medium Access Control Layers for Combined Fixed and Mobile Operation in Licensed Bands," IEEE Computer Society and IEEE Microwave Theory and Techniques Society, Feb. 2006.

[13] TR 25.814, V7.1.0, "Physical Layer Aspects for Evloved Universal Radio Access (UTRA)," 3rd Generation Partnership Project, Technical Specification Group Radio Access Network, Sep. 2006.

[14] I. C. Wong and B. L. Evans, "Optimal Downlink OFDMA Resource Allocation with Linear Complexity to Maximize Ergodic Rates," IEEE Trans. Wireless Commun., vol. 7, pp. 962-971, Feb. 2008.

[15] L. Hoo, B. Halder, J. Tellado, and J. M. Cioffi, "Multiuser Transmit Optimization for Multicarrier Broadcast Channels: Asympotic FDMA capacity region and algorithms," IEEE Trans. Commun., vol. 52, pp. 922-930, Jun. 2004.

[16] Z. Shen, J. G. Andrews, and B. L. Evans, "Adaptive Resource Allocation in Multiuser OFDM Systems With Proportional Rate Constraints," IEEE Trans. Wireless Commun., vol. 4, pp. 2726-2737, Nov. 2005.

[17] S. T. Chung and A. J. Goldsmith, "Degrees of freedom in adaptive modulation: A unified view," IEEE Trans. Commun., vol. 49, pp. 15611571, Sep. 2001.

[18] W. R. Stevens, TCP/IP illustrated, Volume I The protocols. Addison Wesley, Feb. 2000.

[19] B. Veal, K. Li, and D. Lowenthal, "New Methods for Passive Estimation of TCP Round Trip Times," ACM Comp. Commun. Review, vol. 3431, pp. 121-134, 2005.

[20] S. Jaiswal, Measurement in the Middle: Inferring End-End Path Properties and Characteristics of TCP Connections through Passive Measurement. PhD thesis, University of Massachusetts Amherst, Sep. 2005.

[21] Z. Han, Z. Ji, and K. J. R. Liu, "Fair Multiuser Channel Allocation for OFDMA Networks Using Nash Bargaining Solutions and Coalitions," IEEE Trans. Commun., vol. 35, pp. 1366-1375, Aug. 2005. 